\title{
L'agroécologie, un nouveau rapport aux savoirs et à l'innovation
}

\author{
Jean-Marc Meynard* \\ Inra, UMR SAD-APT, bâtiment EGER, campus de Grignon, 78850 Thiverval-Grignon, France
}

Reçu le 14 mars 2017 - Accepté le 30 mars 2017

\begin{abstract}
Résumé - Bien loin d'une simple fusion entre agronomie et écologie, l'agroécologie apparaît comme un projet innovant, qui se positionne à la fois dans le champ des sciences de la nature, des sciences économiques et sociales, de la politique et de l'action. L'agroécologie nous conduit à explorer de nouveaux champs de savoir, aux interfaces entre disciplines, sur les impacts agronomiques des régulations biologiques autant que sur les systèmes socioécologiques. Mais, plus encore, elle nous engage à faire évoluer nos manières de travailler: développer les approches systémiques, en renonçant aux séduisantes simplifications du type «un problème, un intrant $»$; revaloriser les savoirs locaux et les mettre en synergie avec les savoirs scientifiques ; décloisonner l'innovation, en repositionnant l'agriculture au sein des territoires et des systèmes alimentaires ; enfin, favoriser les dynamiques d'apprentissage individuel et collectif, source d'innovation et d'adaptation aux situations locales.
\end{abstract}

Mots clés : régulations biologiques / apprentissages / système alimentaire / approche systémique / transition agroécologique

\begin{abstract}
Agroecology, a new relationship to knowledge and innovation. Far from a simple merger between agronomy and ecology, agroecology is an innovative project, concerning simultaneously the fields of natural sciences, of economic and social sciences, politics and action. Agroecology encourages us to explore new fields of knowledge, at the interface of disciplines, on the agronomical impacts of biological regulations, as far as on the socioecological systems. Yet, it mainly urges us to change our ways of working: develop systemic approach, while giving up attractive simplifications such as "one problem, one input"; value local knowledge, while mixing it with scientific knowledge; open up innovation by positioning agriculture at the heart of territories and food systems; finally promote individual and collective learning, source of innovation and adaptation to local situations.
\end{abstract}

Keywords: biological regulations / learning / food system / systemic approach / agroecological transition

Si le terme « agroécologie» est ancien, son utilisation dans le monde agronomique européen est assez récente, ainsi que le soulignent Wezel et al. (2009). L'agroécologie interpelle les agronomes sur l'intérêt de s'appuyer sur les régulations naturelles de l'agroécosystème, plutôt que sur les intrants, pour assurer la production agricole sans gaspiller les ressources, en particulier celles qui ne sont pas renouvelables. Baser la construction des modes de production sur l'agroécologie, souligne Altieri (1992), c'est diminuer les impacts environnementaux, en réduisant, et si possible en éliminant, les intrants chimiques; c'est renforcer la santé des agroécosystèmes en promouvant des techniques qui renforcent le contrôle naturel des bio-agresseurs; c'est optimiser le métabolisme des agroécosystèmes en organisant le recyclage des nutriments ; c'est améliorer la conservation et la régénération des sols, de la ressource en eau et de la biodiversité. Par ricochet,

\footnotetext{
*Correspondance : jean-marc.meynard@inra.fr
}

l'agroécologie modifie le regard porté sur l'insertion de l'agriculture dans le tissu économique et social: la réduction des intrants chimiques et le recyclage favorisent une autonomie, aussi bien financière que décisionnelle, des producteurs, qui les rend moins dépendants du crédit et des fluctuations du prix des consommations intermédiaires. L'agroécologie constitue de ce fait une voie pour assurer la durabilité d'exploitations ayant peu de ressources financières. Elle ouvre ainsi des pistes prometteuses, pour rendre l'agriculture plus durable, tant aux plans social et économique qu'environnemental (Gliessman, 2007).

Wezel et al. (2009), analysant l'emploi du terme agroécologie dans le monde agronomique, mettent en évidence sa polysémie : Agroécologie (ou agro-écologie) désigne, selon les auteurs, tantôt un domaine scientifique interdisciplinaire, tantôt des pratiques agricoles basées sur la valorisation des régulations naturelles, tantôt un mouvement social de soutien à une petite agriculture autonome. Qui plus est, l'acception 
scientifique du terme est plus ou moins large, depuis (version étroite) «l'appropriation par l'agronomie des concepts et des méthodes de l'écologie et leur application aux écosystèmes anthropisés gérés par l'agriculture et par l'élevage » (Inra, 2010), jusqu'à (version large) «l'écologie des systèmes alimentaires » de Francis et al. (2003), qui enrôle, outre l'agronomie et l'écologie, une partie des sciences économiques et sociales et des sciences de l'alimentation. Cette polysémie, qui a pu parfois dérouter, traduit en fait un foisonnement d'idées et d'initiatives, et engage à renouveler les liens, trop souvent distendus, entre agriculture, alimentation, science, nature et projet politique. Ces liens peuvent être décrits selon trois perspectives :

- de nouveaux champs de connaissance, à l'interface entre différentes disciplines;

- une évolution de la manière de regarder et d'analyser le réel, basée sur des approches systémiques et intégratives ;

- de nouvelles démarches d'innovation, collectives et participatives. Ces trois points constituent la trame de cet article.

\section{Mobiliser de nouveaux champs de connaissance}

Dans un document programmatique commun, l'Inra et le Cirad (2016) explicitent les axes de recherche prioritaires «pour une transition agroécologique». Cette prise de position nous éclaire sur les champs de connaissance que l'agroécologie nous engage à ouvrir, ou tout au moins, à renforcer :

- un premier champ de connaissance d'intérêt majeur concerne les effets de la biodiversité (sauvage, cultivée, élevée) sur les régulations biologiques: "régulations biologiques associées aux mélanges de cultivars et d'espèces, aux plantes de services, aux rotations longues, à l'agroforesterie, au bio-contrôle; interactions biotiques que les plantes et animaux sélectionnés entretiennent avec leur environnement (...)». Après une longue période marquée par la spécialisation des chercheurs sur un nombre de plus en plus restreint d'espèces, qualifiées d'espèces modèles (Bonneuil et Thomas, 2009), l'agroécologie renouvelle l'intérêt pour l'étude d'une diversité d'espèces, des associations et des successions de cultures. La question des méthodes favorisant la transposition des acquis scientifiques des espèces modèles aux autres reste cependant largement à instruire;

- un deuxième champ de connaissance concerne le bouclage des cycles biogéochimiques, au niveau des parcelles, des exploitations agricoles et des territoires. Le document de l'Inra et du Cirad met l'accent sur: «Réduction de l'érosion des sols, recharge des nappes, pertes en nutriments, bouclage des cycles de l'eau et des nutriments, conservation du phosphore, fixation biologique d'azote, stockage de carbone et de nutriments dans les sols, recyclage et valorisation des engrais de ferme». Il faudrait rajouter à cette liste la question du recyclage des effluents urbains. En France, les conséquences, sur le gaspillage de nutriments et les pollutions, de la régression de la polyculture-élevage et de la spécialisation régionale vers les cultures ou l'élevage, sous l'effet de dynamiques économiques de long terme, mériteraient d'être mieux étudiées, pour être prises en considération par les politiques publiques;
- le troisième champ de connaissance concerne le fonctionnement des agroécosystèmes, au niveau des exploitations agricoles, des paysages et des territoires : «Organisation des mosaïques paysagères : parcelles, espaces interstitiels, haies et zones humides, place de l'arbre et de la forêt; nouvelles formes collectives de gouvernance territoriale (...)». Une attention particulière doit être accordée aux compromis entre services écosystémiques, de l'échelle locale à l'échelle globale: «compromis entre les services d'approvisionnement (aliments, fibres, énergie, matériaux...), de régulation (cycle de l'eau, gaz à effet de serre, bio-agresseurs) et les services culturels (valeurs associées) rendus par l'agriculture et la forêt», indique le document des deux organismes de recherche. La place accordée aux échelles du paysage et du territoire, sans être totalement nouvelle, est notable: «L'échelle des ménages et des exploitations est celle de la performance économique (revenu, emploi, vulnérabilité, dépendance). L'échelle des territoires est celle de la conception de nouvelles formes d'organisation, où la notion d'arbitrage pour l'utilisation des ressources revêt une pertinence particulière, tout comme celle de la définition des compromis ». L'évaluation, aux différents niveaux d'échelle, des performances multicritères de l'agriculture et des filières devient indispensable. Le concept de système socioécologique, qui désigne l'articulation, au niveau des territoires, entre systèmes socioéconomiques et systèmes écologiques devient un point d'appui pour penser le développement des pratiques agroécologiques (Duru et al., 2015);

- enfin, un quatrième champ de connaissance est mis en avant par le document: l'étude des systèmes d'innovation et des processus impliqués dans la transition agroécologique. Le document Inra-Cirad souligne l'importance des recherches sur les «outils de formation et d'aide à la décision, les technologies nouvelles pour faciliter l'observation et l'interprétation, les outils spécifiques pour les systèmes agroécologiques (semis sous couvert, mélanges, agroforesterie...), l'organisation des filières, ou les politiques publiques d'accompagnement des transitions, de gestion de la ressource foncière et d'émergence de nouveaux systèmes alimentaires $»$. Cette liste pourrait être complétée par l'analyse des réseaux d'acteurs impliqués dans le changement des systèmes alimentaires et son accompagnement (agriculteurs, consommateurs, industriels de l'alimentation, distributeurs, élus locaux, gestionnaires de ressources, ONG), et des processus d'apprentissage associés. On est ici dans le domaine des sciences sociales, qui, au moins autant que les sciences agronomiques, doivent infléchir leur projet de production de connaissance pour favoriser le développement de l'agroécologie.

L'agroécologie nous invite à réaliser des recherches sur les systèmes alimentaires, et en particulier sur les interdépendances entre production, transformation et alimentation. Bien loin d'accepter comme inéluctable la spécialisation des régions du monde induite par la globalisation des marchés, nous sommes ainsi incités à produire des connaissances sur les politiques favorables à l'autosuffisance alimentaire, sur la relation entre relocalisation des productions et diversification des assolements, sur le développement de synergies entre alimentation locale et dynamique des territoires. Mais ces nouvelles 


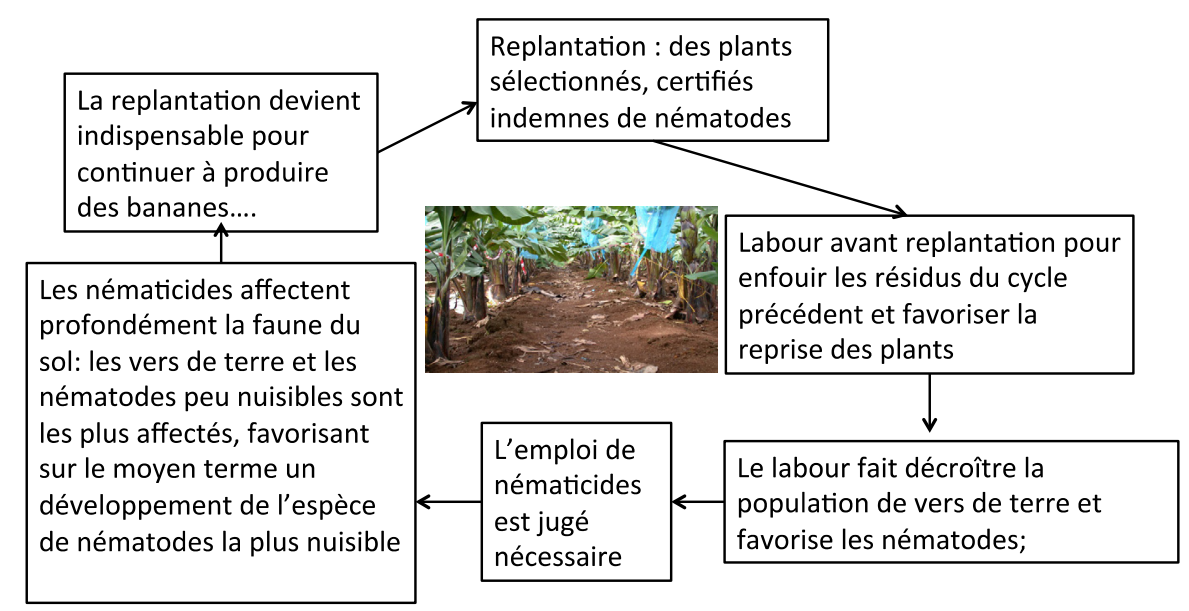

Fig. 1. La vision systémique de l'agroécosytème permet d'imaginer des solutions pour réduire les populations de nématodes et l'usage des nématicides en bananeraie aux Antilles : banane pérenne sans travail du sol? Semis d'une plante d'interculture "piège à nématode»? D'après Clermont-Dauphin et al. (2004).

connaissances seront ineffectives si elles ne sont pas élaborées et mobilisées de manière coordonnée, selon une vision systémique. C'est l'objet du paragraphe suivant.

\section{Adopter une vision systémique}

L'efficacité des intrants, largement employés en agriculture intensive des pays du Nord, a conduit à transformer profondément le raisonnement des pratiques agricoles (Meynard et Girardin, 1991) : à chaque «facteur limitant» est associé un intrant visant à le lever; dans une logique additive, chaque intrant est raisonné indépendamment des autres; le conseil technique s'est centré sur les règles d'utilisation des intrants (quel intrant? quand? à quelle dose?); les organismes de R\&D développent pour cela des «outils d'aide à la décision», qui constituent le cœur de leurs stratégies d'appui technique (Guichard et al., 2017).

L'agroécologie nous invite à adopter un raisonnement différent (Wezel et al., 2014): Réduire les risques de bioagresseurs en amont de l'épidémie et de la parcelle, plutôt que d'intervenir en curatif; valoriser les matières organiques et les éléments minéraux issus de recyclages, avant d'en acheter. Le raisonnement additif laisse place à une approche plus subtile, où l'on prend en compte les interactions entre techniques, et leurs effets indirects. Par exemple, le semis très précoce du colza, sur une parcelle riche en azote, permet de limiter les risques liés aux limaces, au charançon de la tige et aux adventices (Dejoux et al., 2003); autre exemple, une restriction de la densité de semis et de la fertilisation azotée sur tournesol, associée à des variétés très peu sensibles, permet de réduire fortement le développement du phomopsis (Debaeke et al., 2003). Au niveau de la parcelle, la combinaison de plusieurs pratiques à effet indirect permet ainsi de réduire l'usage de pesticides. De même, l'insertion de légumineuses dans les successions ou dans des associations de cultures permet de réduire la fertilisation azotée, sans réduire les marges économiques (Voisin et al., 2014); cependant, le choix de l'association dépend fortement de la période de semis, de la stratégie de désherbage et du mode de commercialisation, comme le montrent Lamé et al. (2015). Dans cette perspective, le raisonnement des pratiques agricoles n'est plus additif, il est systémique (Meynard et al., 2001 ; Doré et al., 2006). Ceci est bien exprimé par la définition de l'itinéraire technique proposée par Sebillotte dès 1974: combinaison logique et ordonnée de techniques.

Un raisonnement systémique des pratiques agricoles s'appuie sur une compréhension intime du fonctionnement de l'agroécosystème, et de ses modalités de pilotage par les agriculteurs, ainsi que l'illustre, par exemple, le cas de la production intensive de bananes aux Antilles (Fig. 1, d'après Clermont-Dauphin et al., 2004). Le mode de production dominant est une monoculture intensive, où les nématodes constituent un problème majeur, justifiant l'emploi fréquent de nématicides (qui polluent sols et eaux) et, au bout de trois ou quatre ans, un arrachage de la bananeraie, trop infestée, suivie d'une replantation de plants certifiés indemnes de nématodes. Un labour avant replantation enfouit les résidus du cycle précédent et affine la structure du sol, favorisant ainsi la reprise des plants. Clermont-Dauphin et al. (2004) montrent que ce labour fait décroître la biomasse de vers de terre, et favorise les nématodes de l'espèce la plus nuisible, Radopholus similis (Cobb). Cet effet du labour sur les nématodes est attribué à la fois à la structure du sol plus aérée, et à la diminution de la population de vers de terre, en position plus élevée sur la chaîne trophique. Les producteurs, constatant les premiers dégâts de nématodes, jugent alors nécessaire l'emploi de nématicides. Ceux-ci affectent encore plus profondément la faune du sol: les vers de terre et les nématodes peu nuisibles sont les plus touchés, favorisant sur le moyen terme un développement de l'espèce de nématodes la plus nuisible. Le système de culture devient alors complètement dépendant des nématicides (trois traitements par an), qui, à moyen terme, ne permettent plus de contrôler les populations de parasites. Le cercle vicieux est bouclé : la replantation devient indispensable! Les solutions pour limiter l'emploi de nématicides doivent rompre ce cercle «monoculture / labour / nématicide» : la banane pérenne (déjà pratiquée par quelques producteurs, sans replantation, sans labour, et sans nématicide); ou la coupure de la monoculture par un an de jachère, ensemencée 


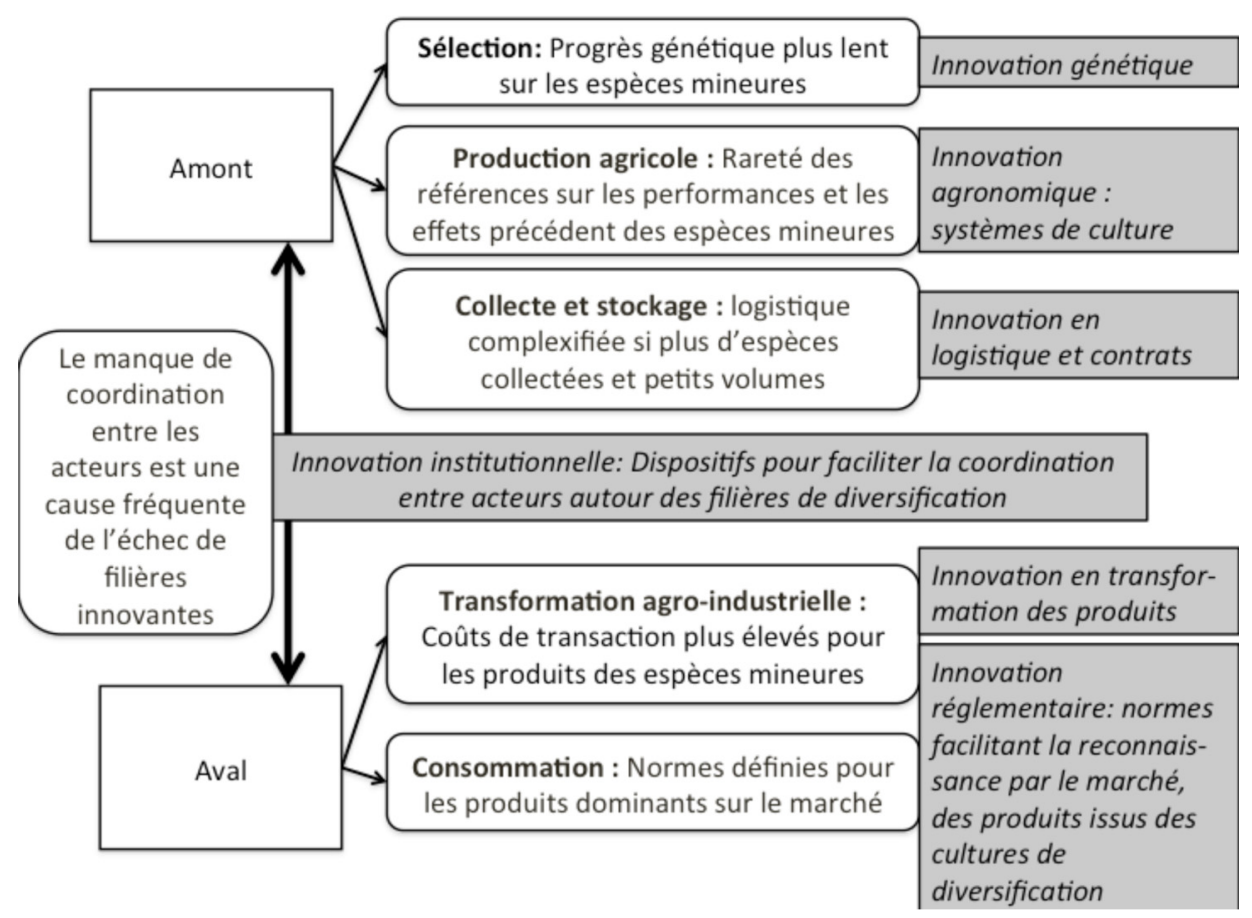

Fig. 2. Représentation schématique des freins au développement des cultures de diversification opérant au niveau du système sociotechnique de l'agriculture française. D'après Meynard et al. (2015).

d'une plante piège à nématodes (la crotalaire). Dans le cadre du Plan banane durable, l'Institut technique tropical, le Cirad et les producteurs de bananes des Antilles françaises se sont engagés dans une démarche de réduction des intrants basée sur cette seconde voie (Dorel et al., 2011).

En mettant en lumière les effets directs, indirects, synergiques ou antagoniques des pratiques, l'approche systémique permet ainsi de concevoir des itinéraires techniques et systèmes de culture substituant à l'emploi d'intrants un pilotage des régulations biologiques. Mais l'intérêt d'une vision systémique ne concerne pas que l'échelle de la parcelle : les solutions durables résultent souvent d'actions à de multiples échelles, parcelle, exploitation agricole, territoire, système alimentaire. L'exemple de la diversification des cultures, élément-clé de la réduction des intrants, montre bien l'importance d'articuler différentes échelles dans l'approche systémique. En France, bien que la diversification soit considérée comme souhaitable par de nombreux acteurs, y compris les pouvoirs publics, un verrouillage sociotechnique autour des espèces majeures entrave le développement des cultures mineures et de leurs filières (Fig. 2, d'après Meynard et al., 2015), et favorise le raccourcissement des rotations. Ce verrouillage implique de nombreux acteurs: les sélectionneurs, qui s'investissent peu sur les espèces mineures, puisque le marché pour leurs semences est par définition limité; les instituts techniques, qui produisent peu de références sur ces effets précédents car ces espèces sont peu cultivées; les chercheurs, qui n'étudient ni l'écophysiologie et la génétique de ces espèces, ni les potentialités industrielles de leurs produits; les agriculteurs, à qui la $\mathrm{R} \& \mathrm{D}$ communique peu d'informations sur ces espèces, et qui ne savent ni comment modifier leurs systèmes de culture pour les introduire, ni comment réagir s'ils obtiennent des résultats décevants; les coopératives et négoces, peu intéressés par les petits volumes de collecte des espèces mineures, qui compliquent leur logistique; l'agro-industrie, qui a calé ses processus de transformation sur les espèces majeures, et apprécie peu les coûts de transaction élevés des espèces mineures; les distributeurs et les consommateurs, qui restent fidèles aux produits issus des espèces dominantes, les atouts de la diversification suscitant peu d'intérêt chez eux. De surcroît, lors de la construction d'une filière nouvelle, basée sur une culture mineure, il est difficile, pour des acteurs qui, souvent, ne se connaissent pas encore, de se coordonner; et le manque de coordination entre les acteurs de l'amont et de l'aval constitue une source fréquente de l'échec de filières innovantes basées sur des cultures de diversification. Il en résulte que les espèces mineures ne se développent pas, même si des débouchés existent potentiellement pour leurs produits (Meynard et al., 2015).

Les stratégies, les activités et les intérêts des différents acteurs étant intimement liés, le verrouillage qui entrave la diversification des cultures est systémique: le déverrouillage doit l'être tout autant (Geels, 2002). Le chemin vers l'agroécologie suppose que les pouvoirs publics et les acteurs concernés mobilisent simultanément et de manière coordonnée plusieurs leviers (Fig. 2):

- des innovations génétiques (intensification de l'effort de sélection sur les petites espèces);

- des innovations agronomiques (rotations longues diversifiées, itinéraires techniques pour les cultures mineures);

- des innovations technologiques (procédés de transformation ouvrant de nouveaux débouchés pour les espèces mineures); 


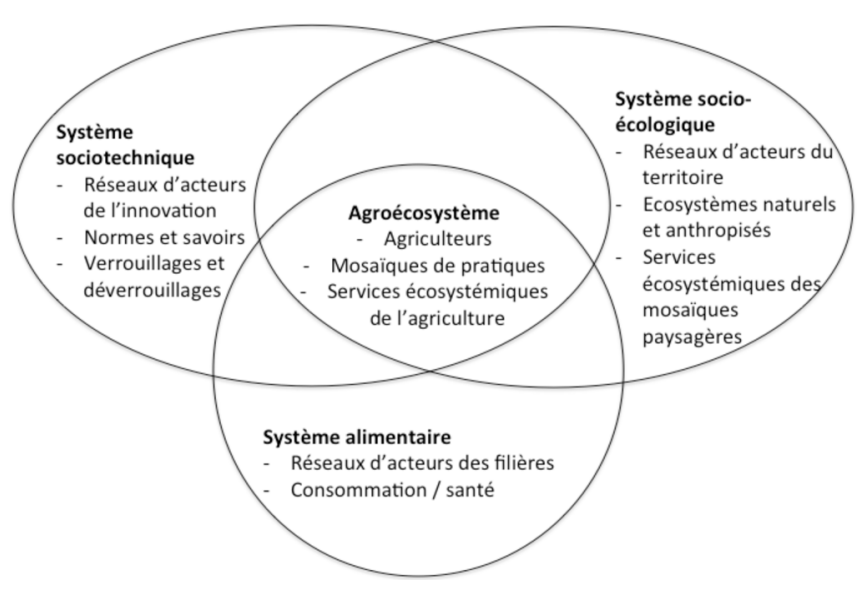

Fig. 3. L'agroécologie, au carrefour des agroécosystèmes, des systèmes socioécologiques, des systèmes sociotechniques et des systèmes alimentaires.

- une évolution des normes marchandes pour aider les marchés à reconnaître l'intérêt (en particulier environnemental) des filières de diversification;

- des dispositifs pour faciliter la coordination entre acteurs des chaînes de valeur.

Il s'agit de choisir, pour chaque question, le(s) système(s) à considérer, sans perdre de vue ses liens avec d'autres systèmes, et ainsi d'agir collectivement à différentes échelles. D'autres exemples du verrouillage sociotechnique de l'agriculture intensive, relatifs à la difficulté de remplacer les pesticides par d'autres moyens de lutte contre les bio-agresseurs, sont donnés par Vanloqueren et Baret (2008), Lamine et al. (2010) et Guichard et al. (2017).

En promouvant une telle vision systémique, l'agroécologie se positionne (Fig. 3) au carrefour entre:

- les agroécosystèmes, où les agriculteurs mettent en œuvre leurs pratiques et produisent différents services écosystémiques ;

- les systèmes socioécologiques (Duru et al., 2015), qui élargissent le panorama à l'ensemble des acteurs du territoire et des écosystèmes présents sur celui-ci ;

- les systèmes sociotechniques (Magrini et al., 2016), qui mettent sous le projecteur les acteurs de l'innovation, les normes et les verrouillages;

- les systèmes alimentaires (Meynard et al., 2016), qui élargissent la vision jusqu'aux acteurs des filières et aux relations entre production, alimentation et santé.

De nombreux champs de savoir sont convoqués par cette vision intégrée, des sciences écologiques aux sciences de la société, en passant par les technologies et les savoirs profanes. Les agronomes-système y sont appelés à jouer, à mon sens, un rôle particulier, grâce à leur familiarité avec les interfaces, et leurs concepts-clés : les pratiques agricoles, les itinéraires techniques et les systèmes de culture ne sont-ils pas déjà au croisement du technique, du social, et de l'écologique?

\section{Innover en s'appuyant sur de nouvelles démarches}

Alors que les systèmes intensifs, basés sur de hauts niveaux d'emploi des intrants de synthèse, visent à homogénéiser le milieu (supprimer les bio-agresseurs, compenser par de l'engrais azoté les écarts de fertilité, apporter de l'eau là où il en manque), l'agroécologie prend comme une donnée la diversité des situations (sols, climat, structures d'exploitations, ressources locales, vulnérabilités écologiques, dynamiques territoriales), et nous propose de nous y adapter. Dans le paradigme agroécologique, la quête de l'innovation universellement efficace, du mode de production idéal, des techniques passe-partout est considérée comme vaine. L'agroécologie contient l'ambition de permettre à chaque agriculteur de concevoir son système de production, de permettre aux acteurs de chaque territoire de s'organiser pour ajuster la gestion des ressources locales, de permettre enfin aux acteurs des systèmes alimentaires d'adapter leurs modes de production, de transformation et de consommation, en fonction de la diversité de leurs préférences. Cette ambition appelle le développement de nouvelles démarches pour innover, impliquant directement les acteurs concernés dans le processus d'innovation, et mobilisant en synergie les savoirs locaux, traditionnels ou acquis par expertise, et les savoirs scientifiques les plus pointus. Ces nouvelles démarches d'innovation privilégient les apprentissages et les concertations, et sont appelées à donner une place croissante à l'articulation entre production et alimentation, trop souvent considérées de manière indépendante.

\subsection{Accompagner le changement et les apprentissages en agriculture}

Les agronomes ont développé différentes démarches de conception de systèmes de culture ou de production innovants, décrites par Meynard et al. (2012). De nombreux exemples de modes de production basés sur les principes de l'agroécologie, et en rupture par rapport à l'agriculture actuelle, ont été proposés dans la littérature scientifique et technique (voir par exemple les synthèses de Viaux 2013, Jacquet et al., 2011; Deguine et al., 2016, Husson et al., 2015). Ces «systèmes innovants » peuvent aider à convaincre des agriculteurs qu'il est possible de changer de logique de production, et leur servir de sources d'inspiration, pour inventer leurs propres systèmes. Mais le changement de mode de production dans une exploitation agricole ne consiste généralement pas en une importation directe d'un système exogène : le changement est progressif, il suit un cheminement spécifique de l'exploitation, et s'accompagne d'apprentissages nombreux (Chantre et al., 2014; Coquil et al., 2009). Toffolini et al. (2016) ou Meynard et Dourmad (2014) ont tenté de spécifier les étapes de ce processus, et de préciser ses conditions de réussite. Ainsi que l'écrivent ces derniers, «la conception débute par un diagnostic (le système de production peut-il être jugé satisfaisant ?). Ce diagnostic permet d'identifier les points clés à améliorer (quels indicateurs ne sont pas à un niveau satisfaisant? quels processus sont en jeu ?), et d'imaginer les changements de pratiques susceptibles d'améliorer la situation. Après mise en œuvre de ces changements, un nouveau 
diagnostic est réalisé, qui permet de vérifier si la situation a été améliorée, et de déterminer de nouvelles priorités : c'est une véritable boucle d'amélioration continue qui se met en route ». Le changement est favorisé par l'accompagnement d'un conseiller, qui aide l'agriculteur à réaliser ses diagnostics et l'alimente en solutions exogènes. L'agriculteur met ainsi au point, année après année, son nouveau système, en même temps qu'il apprend à le piloter, se convainc de son intérêt, et réorganise son travail, ses moyens de production et ses débouchés (Mischler et al., 2009).

Les conditions de la réussite d'une telle démarche de reconception des systèmes agricoles, basée sur les principes de l'agroécologie ont été précisées par différents auteurs:

- une bibliothèque d'innovations (variétés, outils d'aide à la décision, méthodes de protection intégrée, méthodes de gestion des matières organiques, cultures de diversification...). Il est important que chaque innovation de cette bibliothèque soit caractérisée de manière précise, pour aider l'agriculteur dans ses choix : matériel et compétences nécessaires, impacts attendus sur l'environnement et sur la production, synergies avec d'autres pratiques, conditions de réussite... (Meynard, 2012). Cette bibliothèque peut être alimentée par les agronomes, mais aussi par les praticiens (Salembier et al., 2016, Guichard et al., 2015);

- la mise en œuvre de boucles d'apprentissage basées sur des critères de performance renouvelés (incluant par exemple la réduction des intrants, ou le recyclage des nutriments). Toffolini et al. (2016) montrent que les agriculteurs utilisent de très nombreux indicateurs dans leurs apprentissages, sur lesquels peu de recherches ont été menées ;

- la mise en place de dynamiques collectives (groupes d'agriculteurs innovants): les travaux des sociologues (Darré, 1994; Warner, 2007; Lamine 2011) montrent le rôle des groupes d'échange entre agriculteurs dans l'apprentissage des systèmes innovants, à la fois source d'idées et de démultiplication des expériences, et vecteur de réassurance face à la prise de risque.

\subsection{Organiser des concertations entre acteurs des territoires}

Le changement au niveau d'exploitations agricoles individuelles est évidemment insuffisant pour gérer des ressources collectives, comme l'eau d'irrigation, la biodiversité ou un captage d'eau potable. À l'échelle du paysage, c'est de la concertation entre les acteurs concernés qu'émergera la solution (Benoît et al., 2012). Comment cette concertation peut-elle être organisée, pour innover collectivement, alors qu'au départ, les acteurs ne sont parfois d'accord ni sur leurs attentes, ni sur leurs représentations de la situation, ni même sur les bénéfices qu'ils pourraient tirer d'une coordination?

Duru et al. (2015) proposent une méthodologie participative en cinq étapes pour favoriser la transition agroécologique de l'agriculture au niveau des territoires :

- analyse-diagnostic de la situation actuelle;

- identification des «drivers » du changement, c'est-à-dire des évolutions extérieures au territoire qui conditionnent son futur;

- conception de systèmes de culture et d'organisations paysagères pour une agriculture basée sur la biodiversité ;
- proposition d'étapes permettant de passer de la situation actuelle à une agriculture agroécologique ;

- conception de structures de gouvernance et de stratégies de gestion collective destinées à guider la transition.

La démarche du groupe ComMod (Barreteau et al., 2003, Etienne, 2010) s'adresse en priorité à la gestion collective de ressources territoriales. Elle peut être décrite par trois étapes :

(i) la construction d'une représentation partagée des processus territoriaux qu'il s'agit de piloter; les parties prenantes développent ensemble un modèle informatique qui rend compte des interactions clés entre les systèmes écologiques, agricoles et sociaux;

(ii) un jeu de rôles, qui, en mettant les acteurs en situation, leur permet d'appréhender les conséquences (simulées par le modèle) de leurs choix individuels ou des scénarios de gestion collective qu'ils imaginent;

(iii) un débriefing du jeu de rôle, qui débouche sur des apprentissages collectifs concernant les limites des stratégies adoptées, et sur la mise en débat de règles de gestion collective susceptibles de mieux donner satisfaction à tous, qui seront testées au tour de jeu suivant (retour au (ii)). Berthet et al. (2014), qui s'intéressent à la conception de mosaïques paysagères favorables à la biodiversité, suggèrent de désamorcer d'éventuels conflits entre différents acteurs (les uns privilégiant la production, les autres privilégiant d'autres services écosystémiques), en considérant la ressource à gérer comme un « inconnu commun »: les acteurs seront d'autant plus ouverts à des solutions originales que le bien commun à gérer ne sera pas défini au départ, mais le résultat d'une construction collective.

En mettant en exergue la dimension territoriale des processus écologiques, l'agroécologie appelle ainsi à des arrangements collectifs, trop souvent négligés par l'agriculture intensive (Wezel et al., 2016). Le diagnostic partagé de Duru et al. (2015), la construction participative du modèle de Commod, la construction simultanée du bien à gérer et des modes de gestion de Berthet et al. (2014), sont autant de propositions stimulantes pour amener les acteurs à dépasser leurs oppositions, et à instruire en collectif la solution du problème commun.

\subsection{Concevoir des innovations couplées entre agriculture et alimentation}

Meynard et al. (2016) montrent que, pour améliorer la durabilité des systèmes alimentaires, il est souhaitable de s'affranchir du mode d'innovation le plus courant, qui consiste à adapter les systèmes de culture aux exigences de l'aval. Ces auteurs proposent d'orienter le processus de conception vers des « innovations couplées » (c'est-à-dire étroitement coordonnées) entre production et alimentation. L'agroécologie, en tant qu'écologie des systèmes alimentaires, appelle donc une réorganisation des dispositifs et des procédures de conception des innovations: élargissement du collectif d'acteurs impliqués, réalisation d'un diagnostic partagé sur les points clés qu'il sera nécessaire d'améliorer pour accroître la durabilité des systèmes alimentaires ; création de dispositifs 
interinstitutionnels de partage des objectifs et des concepts d'innovation.

L'innovation couplée repose sur un collectif d'acteurs dont les contours ne sont pas aisés à définir: Duru et al. (2015) ou Wezel et al. (2016) voient les territoires locaux comme les lieux privilégiés de conduite des processus d'innovation pour la transition agroécologique. Mais, dans le cas du déverrouillage pour la diversification des cultures, on a vu que si certains processus d'innovation relèvent bien d'un niveau local (systèmes de culture), d'autres n'ont de sens qu'à des échelles plus larges: il en est ainsi, par exemple, de la sélection de variétés pour les espèces orphelines, ou de la conception du cadre de soutien aux dispositifs de partenariat, qui relèvent plutôt du niveau national. Ainsi, une articulation entre processus de conception à différentes échelles, entre concepteurs locaux et nationaux, apparaît indispensable. Dans ces conditions, comment les concepteurs doivent-ils s'organiser pour partager les buts et les concepts innovants nécessaire au couplage des innovations?

La coordination des processus de conception initiés par ces différents acteurs suppose d'abord un partage de la vision de l'évolution du système alimentaire. Or, comme le soulignent Stuart et Worosz (2012), les acteurs dominants ont tendance à imposer aux autres leur vision du système alimentaire, souvent inféodée à leurs intérêts. Cet état de fait est entretenu par l'absence de méthode reconnue permettant la réalisation de diagnostics des systèmes alimentaires. Meynard et al. (2016) proposent de mettre au point une telle méthode, visant à identifier les sources de problèmes et les ressources qui peuvent être mobilisées pour les résoudre. Un tel diagnostic pourrait s'inspirer des propositions d'Ostrom (2007), pour le diagnostic des systèmes socioécologiques et de Doré et al. (1997) pour le diagnostic agronomique régional.

Différents dispositifs interinstitutionnels de partage des objectifs et des concepts d'innovation sont proposés dans la littérature, et mériteraient d'être mis à l'épreuve pour favoriser l'innovation couplée au sein des systèmes alimentaires. Par exemple, Bos et al. (2009) proposent de conduire des ateliers de conception focalisés sur les besoins basiques des différents acteurs du système alimentaire, plutôt que sur leurs intérêts à court terme, dépendants du contexte, et plus sujets à des oppositions irréductibles. Le Masson et al. (2012) mettent en avant l'intérêt d'une «plateforme d'animation de l'écosystème des concepteurs », où chercheurs, concepteurs de différentes entreprises et sous traitants échangent sur les solutions innovantes imaginées par chacun, et ce faisant spécifient ensemble les concepts sous-jacents, explorent ensemble d'autres concepts, analysent la compatibilité des concepts entre eux... À l'opposé, certaines manières d'organiser la coordination entre acteurs sont peu favorables à l'innovation couplée: Ainsi, les «référentiels de bonnes pratiques agricoles » (obligation de moyens), souvent imposés aux agriculteurs par les entreprises d'aval pour maîtriser leurs sources d'approvisionnement sont-elles contre-productives, car l'agriculteur, considéré comme un exécutant, n'est pas source d'innovation (de Sainte Marie, 2014; Meynard et al., 2012). Un partenariat basé sur une obligation de résultats laisse au contraire la porte ouverte à la conception, par l'agriculteur, de solutions techniques originales pour répondre aux attentes de son client. $\mathrm{Si}$, comme dans le cas de la filière «lin oléagineux pour l'alimentation animale» (label
«Bleu-Blanc-Cœur»), cette obligation de résultat est mise en débat dans une structure de concertation interne à la filière émergente, les difficultés rencontrées par les agriculteurs peuvent déboucher sur des innovations à d'autres niveaux de la filière (évolution du barème de prix, organisation de la collecte), et sur des apprentissages collectifs (Meynard et al., 2015).

\section{Conclusion}

Bien loin, comme pourrait le laisser croire son étymologie, d'une fusion entre agronomie et écologie, l'agroécologie apparaît comme un projet innovant, qui se positionne à la fois dans le champ des sciences de la nature, des sciences économiques et sociales, de la politique et de l'action. Relier agriculture, alimentation, science, milieu naturel et projet politique, quelle belle ambition!

Face à la diversité des situations agricoles, l'agroécologie nous aide à imaginer et mettre au point une diversité de solutions. Ces solutions sont souvent basées sur des innovations locales, conçues aux échelles de la parcelle ou du territoire, mais elles se combinent avec des innovations issues d'acteurs nationaux ou internationaux. Elles reposent sur un dialogue, des concertations et des collaborations entre les acteurs de l'agriculture, des territoires et des systèmes alimentaires. L'agroécologie nous conduit à explorer de nouveaux champs de savoir, aux interfaces entre disciplines, sur les impacts agronomiques des régulations biologiques ou sur les systèmes socioécologiques. Mais elle nous engage surtout à faire évoluer nos manières de travailler: développer les approches systémiques, en renonçant aux séduisantes simplifications du type « un problème, un intrant »; revaloriser les savoirs locaux et les mettre en synergie avec les savoirs scientifiques; décloisonner l'innovation, en repositionnant l'agriculture au sein des territoires et des systèmes alimentaires; enfin, réapprendre à apprendre, en développant les dynamiques d'apprentissage, individuel et collectif, source d'adaptation aux situations locales, autant que d'innovation.

\section{Références}

Altieri MA. 1992. Agroecological foundations of alternative agriculture in California. Agric Ecosyst Environ 39: 23-53. doi:10.1016/0167-8809(92)90203-n.

Barreteau O, Antona M, d'Aquino P, et al. 2003. Our companion modelling approach. J Artif Soc Soc Simul 6 (2): 1. Disponible sur http://jasss.soc.surrey.ac.uk/6/2/1.html.

Benoît M, Rizzo D, Marraccini E, et al. 2012. Landscape agronomy: a new field for addressing agricultural landscape dynamics. Landsc Ecol 27: 1385-1394.

Berthet ET, Bretagnolle V, Segrestin B. 2014. Surmonter un blocage de l'innovation par la conception collective. Cas de la réintroduction de luzerne dans une plaine céréalière. Fourrages 217: 13-21.

Bonneuil C, Thomas F. 2009. Gènes, pouvoirs et profits. Recherche publique et régimes de production des savoirs de Mendel aux OGM. Paris : Quae, 619 p.

Bos AP, Groot Koerkamp PWG, Gosselink JMJ, Bokma SJ. 2009. Reflexive interactive design and its application in a project on sustainable dairy husbandry systems. Outl Agric 38: 137-145. Available from http://doi.org/10.5367/000000009788632386. 
Chantre E, Cerf M, Le Bail M. 2014. Transitional pathways towards input reduction on French field crop farms. Int J Agric Sustain 13: 69-86. doi:10.1080/14735903.2014.945316.

Clermont-Dauphin C, Cabidoche YM, Meynard JM. 2004. Effects of intensive monocropping of bananas on properties of volcanic soils in the uplands of the French West Indies. Soil Use Manag 20 (2): $105-113$.

Coquil X, Blouet A, Fiorelli JL, Bazard C, Trommenschlager JM. 2009. Conception de systèmes laitiers en agriculture biologique : une entrée agronomique. Inra Prod Anim 22: 221-234.

Darré JP, ed. 1994. Pairs et experts dans l'agriculture. Dialogues et production de connaissances pour l'action. Ramonville SaintAgne, France : Éditions Erès.

Debaeke P, Estragnat A, Reau R. 2003. Influence of crop management on sunflower stem canker (Diaporthe helianthi). Agronomie 23(7): 581-592.

Deguine JP, Gloanec C, Laurent JP. 2016. Protection agroécologique des cultures. Versailles : Éditions Quae, 287 p.

Dejoux JF, Meynard JM, Reau R, Roche R, Saulas P. 2003. Evaluation of environment friendly crop management systems for winter rapeseed based on very early sowing dates. Agronomie 23: 725736.

Doré T, Sebillotte M, Meynard JM. 1997. A diagnostic method for assessing regional variation in crop yield. Agric Syst 54: 169-188.

Doré T, Le Bail M, Martin P, Ney B, Roger-Estrade J. 2006. L'agronomie aujourd'hui. Versailles : Éditions Quae, 367 p.

Dorel M, Tixier P, Dural D, Zanoletti S. 2011. Alternatives aux intrants chimiques en culture bananière. Innov Agron 16: 1-11.

Duru M, Thérond O, Fares M. 2015. Designing agroecological transitions. Agron Sustain Dev 35: 1237-1257. doi:10.1007/ s13593-015-0318-x.

Etienne M, ed. 2010. Companion modelling. A participatory approach to sustainable development. Versailles : Éditions Quae.

Francis C, Lieblein G, Gliessman S, et al. 2003. Agroecology: the ecology of food systems. J Sustain Agric 22: 99-118. Available from http://doi.org/10.1300/J064v22n02_07.

Geels FW. 2002. Technological transitions as evolutionary reconfiguration processes: a multilevel perspective and a case-study. Res Pol 31: 1257-1274. Available from http://doi.org/10.1016/ s0048-7333(02)00062-8.

Gliessman SR. 2007. Agroecology: ecological processes in sustainable agriculture, 2nd ed. Boca Raton: Lewis Publisher (CRC Press), 408 p. doi:10.1017/S0014479707005364.

Guichard L, Ballot R, Halska J, et al. 2015. AgroPEPS, un outil web collaboratif de gestion des connaissances pour produire, échanger, pratiquer, s'informer sur les systèmes de culture durables. Innov Agron 43: 83-94.

Guichard L, Dedieu F, Jeuffroy M-H, Meynard J-M, Reau R, Savini I. 2017. Le plan Ecophyto de réduction d'usage des pesticides en France : décryptage d'un échec et raisons d'espérer. Cah Agric 26: 14002. doi:10.1051/cagri/2017004.

Husson O, Tran Quoc H, Boulakia S, et al. 2015. Co-designing innovative cropping systems that match biophysical and socioeconomic diversity. The DATE approach to conservation agriculture in Madagascar, Lao PDR and Cambodia. Renew Agric Food Syst 31: 452-470. doi:10.1017/S174217051500037X.

Inra. 2010. Document d'orientation 2010-2020, une science pour l'impact. Disponible sur http://inra.dam.front.pad.brainsonic. com/ressources/afile/224599-e325e-resource-document-d-orien tation-2010-2020.html.

Inra, Cirad. 2016. Agro-écologie : le positionnement des recherches de l'Inra et du Cirad. Disponible sur https://inra-dam-frontresources-cdn.brainsonic.com/ressources/afile/373359-42327- resource-agro-ecologie-positionnement-des-recherches-inra-etcirad-2016.pdf.

Jacquet F, Butault JP, Guichard L. 2011. An economic analysis of the possibility of reducing pesticides in French field crops. Ecol Econ 70: 1638-1648. doi:10.1016/j.ecolecon.2011.04.003

Lamé A, Jeuffroy MH, Pelzer E, Meynard JM. 2015. Les agriculteurs sources d'innovations: exemple des associations pluri-spécifiques dans le grand Ouest de la France. Agron Environ Soc 5 (2).

Lamine C. 2011. Transition pathways towards a robust ecologization of agriculture and the need for system redesign. Cases from organic farming and IPM. J Rural Stud 27: 209-219. doi:10.1016/ j. jrurstud.2011.02.001.

Lamine C, Meynard JM, Bui S, Messéan A. 2010. Réductions d'intrants: des changements techniques, et après? Effets de verrouillage et voies d'évolution à l'échelle du système agrialimentaire. Innov Agron 8: 121-134.

Le Masson P, Weil B, Hatchuel A, Cogez P. 2012. Why aren't they locked in waiting games? Unlocking rules and the ecology of concepts in the semiconductor industry. Tech Anal Strat Manag 24 (6): 617-630. Available from http://doi.org/10.1080/ 09537325.2012 .693675$.

Magrini MB, Anton M, Cholez C, et al. 2016. Why are grain-legumes rarely present in cropping systems despite their environmental and nutritional benefits? Analyzing lock-in in the French agrifood system. Ecol Econ 126: 152-162. Available from http://doi.org/ 10.1016/j.ecolecon.2016.03.024.

Meynard JM. 2012. Innovating in cropping and farming systems (chapter 5). In: Coudel E, Devautour H, Soulard CT, Faure G, Hubert B, eds. Renewing innovation systems in agriculture and food: how to go towards more sustainability? Wageningen Academic Publishers, pp. 89-108.

Meynard JM, Girardin P. 1991. Produire autrement. Courr Environ 15: 1-19. Disponible sur http://www7.inra.fr/lecourrier//wp-content/ uploads/2012/01/C15Girardin.pdf.

Meynard JM, Doré T, Habib R. 2001. L'évaluation et la conception de systèmes de culture pour une agriculture durable. C R Acad Agric Fr 87(4): 223-236.

Meynard JM, Dedieu B, Bos AP. 2012. Re-design and co-design of farming systems. An overview of methods and practices. In: Darnhofer I, Gibons D, Dedieu B, eds. Farming systems research into the 21st century: the new dynamic. Springer, pp. 407-432.

Meynard JM, Dourmad JY. 2014. L'innovation en élevage : de nouvelles démarches pour de nouveaux enjeux. In : Ingrand $\mathrm{S}$, Baumont R, eds. Quelles innovations pour quels systèmes d'élevage ? Inra Prod Anim 27, numéro spécial.

Meynard JM, Messéan A, Charlier A, et al. 2015. La diversification des cultures : lever les obstacles agronomiques et économiques. Versailles : Éditions Quae, 106 p.

Meynard JM, Jeuffroy MH, Le Bail M, Lefèvre A, Magrini MB, Michon C. 2016. Designing coupled innovations for the sustainability transition of agrifood systems. Agric Syst. Available from http://doi.org/10.1016/j.agsy.2016.08.002.

Mischler P, Lheureux S, Dumoulin F, et al. 2009. En Picardie, 8 fermes de grande culture engagées en production intégrée réduisent fortement les pesticides sans baisse de marge. Courr Environ 57: 73-91.

Ostrom E. 2007. A diagnostic approach for going beyond panaceas. PNAS 104(39): 15181-15187. Available from http://doi.org/ 10.1073/pnas.0702288104.

de Sainte Marie C. 2014. Rethinking agri-environmental schemes. A result-oriented approach to the management of species-rich grasslands in France. J Environ Plan Manag 57(5): 704-719. doi:10.1080/09640568.2013.763772. 
Salembier C, Elverdin JH, Meynard JM. 2016. Tracking on-farm innovations to unearth alternatives to the dominant soybean-based system in the Argentinean Pampa. Agron Sustain Dev 36.

Stuart D, Worosz MR. 2012. Risk, anti-reflexivity, and ethical neutralization in industrial food processing. Agric Hum Values 29: 287-301. Available from http://doi.org/10.1007/ s10460-011-9337-7.

Toffolini Q, Jeuffroy MH, Prost L. 2016. Indicators used by farmers to design agricultural systems: a survey. Agron Sustain Dev 36: 5. doi:10.1007/s13593-015-0340-z.

Vanloqueren G, Baret P. 2008. Why are ecological, low-input, multiresistant wheat cultivars slow to develop commercially? A Belgian agricultural 'lock-in' case study. Ecol Econ 66: 436-446. doi:10.1016/j.ecolecon.2007.10.007.

Viaux P. 2013. Systèmes intégrés : une troisième voie en grande culture, $2^{\mathrm{e}}$ éd. Éditions La France Agricole, coll. " Agri production ».
Voisin AS, Guéguen J, Huyghe C, et al. 2014. Legumes for feed, food, biomaterials and bioenergy in Europe: a review. Agron Sustain Dev 343: 361-380.

Warner KD. 2007. Agroecology in action; extending alternative agriculture through social networks. London, UK: The MIT Press.

Wezel A, Bellon S, Doré T, Francis C, Vallod D, David C. 2009. Agroecology as a science, a movement and a practice. A review. Agron Sustain Dev 29: 503-515. doi:10.1051/agro/2009004.

Wezel A, Casagrande M, Celette F, Vian JF, Ferrer A, Peigné J. 2014. Agroecological practices for sustainable agriculture. A review. Agron Sustain Dev 14: 1-20. doi:10.1007/s13593-013-0180-7.

Wezel A, Brives H, Casagrande M, Clément C, Dufour A, Vandenbroucke P. 2016. Agroecology territories: places for sustainable agricultural and food systems and biodiversity conservation. Agroecol Sustain Food Syst 40(2): 132-144. doi:10.1080/21683565.2015.1115799.

Citation de l'article : Meynard J-M. 2017. L'agroécologie, un nouveau rapport aux savoirs et à l'innovation. OCL 24(3): D303. 